\title{
Le site du monastère d'Annegray (Haute-Saône) : les prospections géophysiques
}

Sébastien Bully, Emmet Marron, Roseanne Schot, Gerard Dowling et Laurent Fiocchi

\section{Q OpenEdition \\ 12 Journals}

\section{Édition électronique}

URL : https://journals.openedition.org/cem/11856

DOI : $10.4000 /$ cem. 11856

ISSN : 1954-3093

Éditeur

Centre d'études médiévales Saint-Germain d'Auxerre

Édition imprimée

Pagination : 9-15

ISSN : 1623-5770

Référence électronique

Sébastien Bully, Emmet Marron, Roseanne Schot, Gerard Dowling et Laurent Fiocchi, « Le site du monastère d'Annegray (Haute-Saône) : les prospections géophysiques », Bulletin du centre d'études médiévales d'Auxerre | BUCEMA [En ligne], 15 | 2011, mis en ligne le 05 octobre 2011, consulté le 22 septembre 2022. URL : http://journals.openedition.org/cem/11856 ; DOI : https://doi.org/10.4000/ cem. 11856

Ce document a été généré automatiquement le 22 septembre 2022.

\section{(c)}

Creative Commons - Attribution - Pas d'Utilisation Commerciale - Partage dans les Mêmes Conditions 4.0 International - CC BY-NC-SA 4.0

https://creativecommons.org/licenses/by-nc-sa/4.0/ 


\title{
Le site du monastère d'Annegray (Haute-Saône) : les prospections géophysiques
}

\author{
Sébastien Bully, Emmet Marron, Roseanne Schot, Gerard Dowling et \\ Laurent Fiocchi
}

\section{Présentation}

1 Le hameau d'Annegray, sur la commune de la Voivre, est traditionnellement identifié comme le site du monastère d'Anagrates; la découverte des fondations de l'église romane Saint-Jean-Baptiste et de sarcophages mérovingiens par le docteur Cugnier en 1958 participe à cette identification ${ }^{1}$. Annegray est situé dans la vallée supérieure du Breuchin, pénétrant les premiers contreforts méridionaux du massif vosgien. Le monastère était également à proximité de la cluse de Faucogney, verrouillant la route en direction du col du Mont de Fourche et de la vallée de la Moselle. Ce site est considéré traditionnellement comme la première fondation sur le continent du grand moine irlandais, à la fin $\mathrm{du} \mathrm{VI}^{\mathrm{e}}$ siècle. Le récit de la Vie de saint Colomban, écrit par Jonas de Bobbio dans les années 640, relate dans ces termes le choix de l'emplacement du premier établissement: «(...) il y avait alors une vaste solitude appelée Vôge où se trouvait un castrum ruiné que la tradition des anciens nommait Anagrates. Il s'y établit avec les siens $(. . .)^{2}{ }^{\prime}$.

2 L'un des enjeux principal des recherches - en parallèle avec celles menées sur Luxeuil est d'essayer de déterminer la forme que prit ce monachisme irlandais lors de son installation en Gaule, entre conservatisme d'usages insulaires et adaptation aux données locales. En particulier, le parcellaire cadastral d'Annegray conserve un certain nombre d'enclos ovoïdes, dont on envisage prudemment, depuis longtemps, que certains trouvent leurs origines dans ces clôtures circulaires, qui sont l'une des caractéristiques du monachisme irlandais. C'est le cas, plus particulièrement, de la 
circonvolution formant une butte au sommet de laquelle ont été découverts les vestiges de l'église et les tombes (fig. 1).

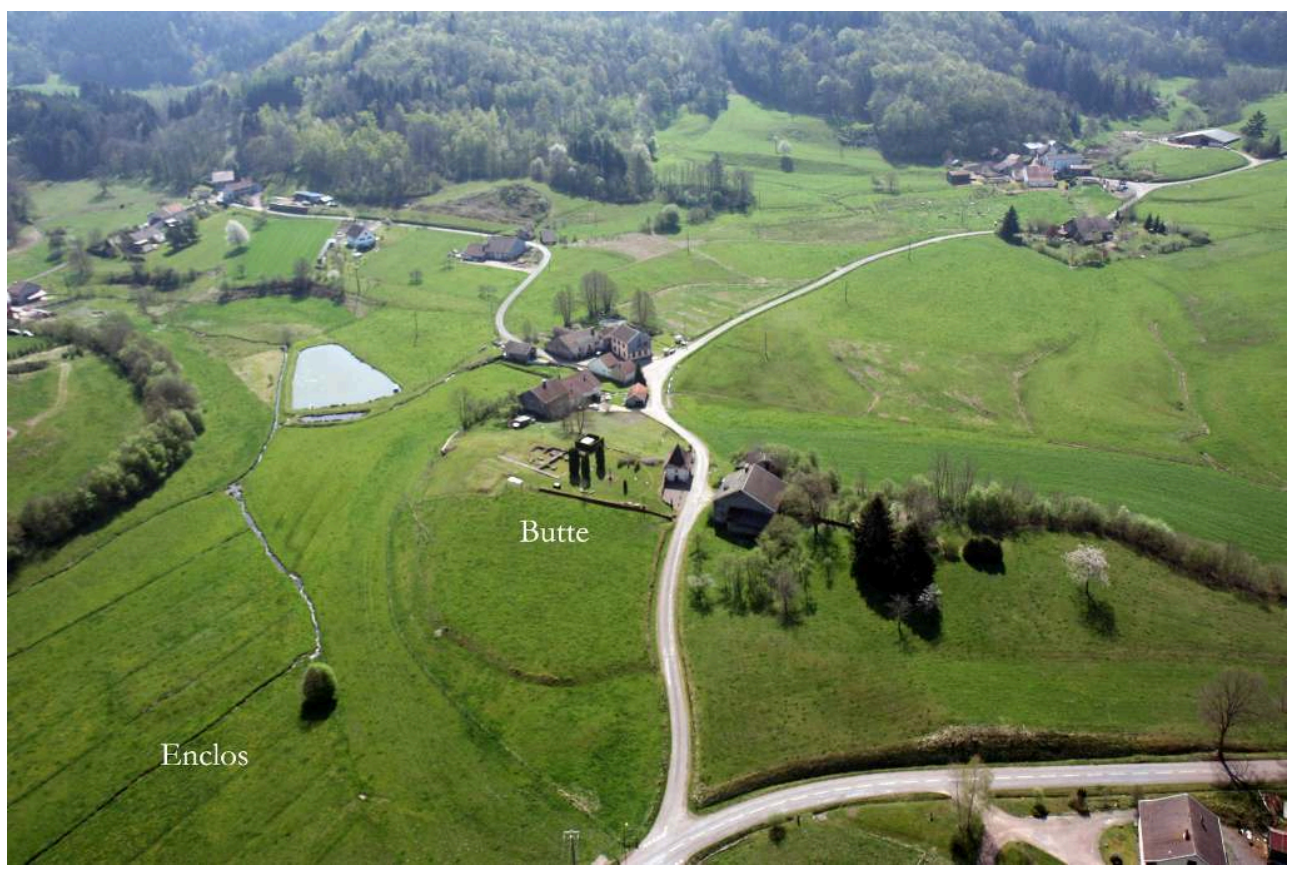

Fig. 1 - Annegray, vue générale du site depuis le nord (cl. Amis de saint Colomban).

Durant cette première campagne de deux semaines, nous avons donc fait le choix de prospecter prioritairement la butte accueillant les vestiges et un champ la bordant au nord (Breuil d'Annegray). C'est dans ce champ que des structures circulaires avaient été observées depuis le point de vue dominant de l'église Saint-Martin de Faucogney ${ }^{3}$. La surface étudiée a été divisée en trois secteurs, représentant un peu plus de 4 hectares. Seul le secteur $\mathrm{n}^{\circ} 1$, au nord de la butte, a révélé des vestiges et fait par conséquent l'objet d'une description. La méthode géophysique mise en œuvre à Annegray est celle de la magnétométrie à haute résolution; les instruments utilisés sont deux Bardington Crad 601, l'un à gradiomètre unique et l'autre à gradiomètre double (fig. 2). Dans le même temps, nous avons dressé un relevé au tachéomètre laser des vestiges de l'église Saint-Jean-Baptiste et un relevé topographique du site. 


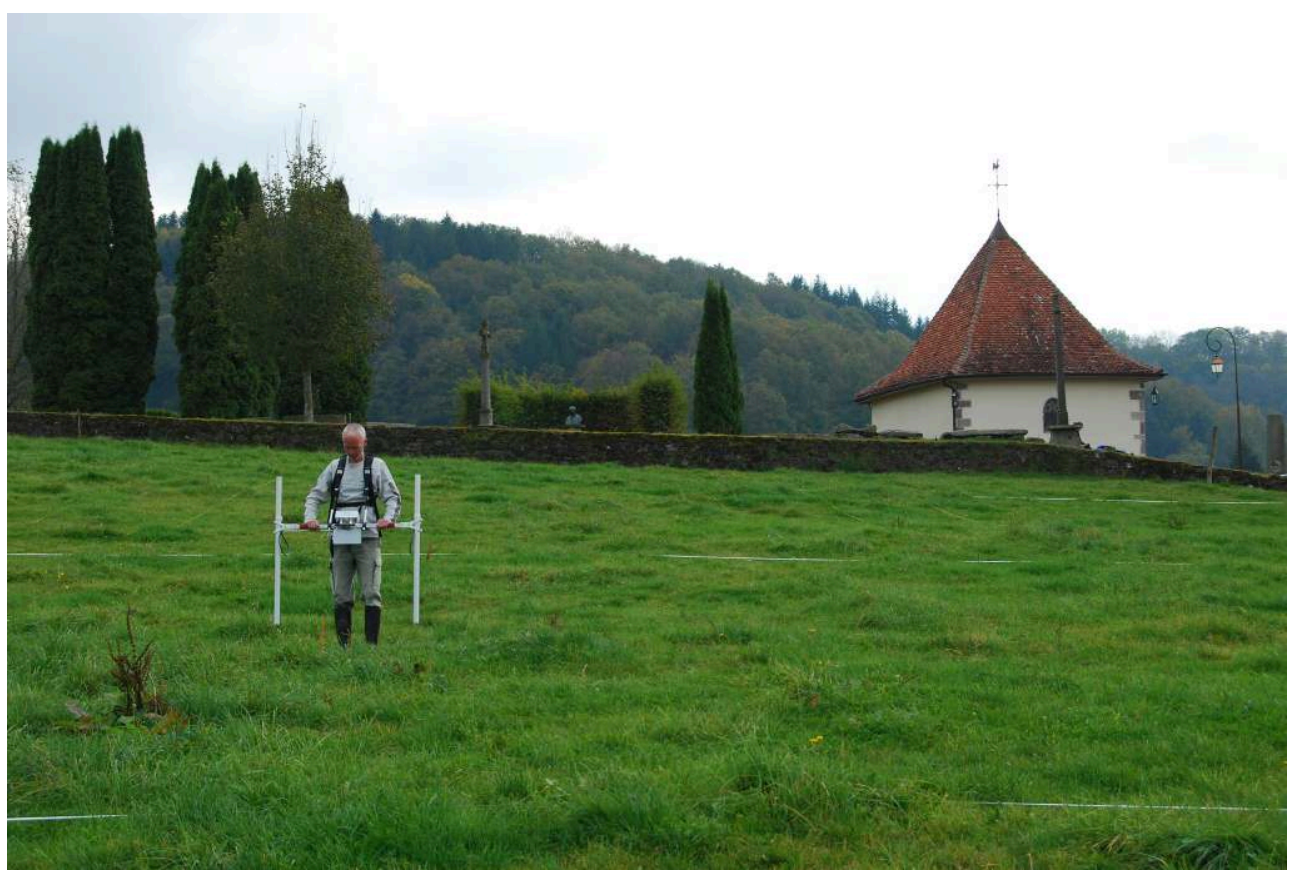

Fig. 2 - Annegray, prospection géophysique sur le flanc nord de la butte (cl. S. Bully).

4 L'ensemble de ces travaux s'inscrit dans le projet collectif de recherche « Monastère en Europe occidentale $\left(v^{e}-x^{e}\right.$ siècle) $»-$ présenté dans ce même numéro - abondé par le projet FABER du conseil régional de Bourgogne et porté par l'UMR Artehis ${ }^{4}$. La prospection a également été réalisée dans le cadre d'une collaboration engagée avec le département d'archéologie de l'université de Galway (Irlande).

\section{Description}

Le secteur $\mathrm{n}^{\circ} 1$ recouvre le quart nord-est de la butte et le champ en contrebas. Il s'inscrit dans un rectangle mesurant environ $180 \times 180 \mathrm{~m}$ dans ses dimensions maximales; sa surface est de 2,7 hectares. Le champ du Breuil d'Annegray est une pâture humide traversée par de longues tranchées de drainages orientées nord-sud et collectées dans un petit ruisseau (naturel ?), qui suit une ligne nord-est - sud-ouest. Lors de fortes pluies, drains et ruisseaux débordent et inondent une grande partie du champ. C'est pourtant dans ce secteur humide et apparemment peu propice à la construction (aujourd'hui), que l'on a repéré la structure archéologique la plus importante, au pied de la butte, à une soixantaine de mètres au nord des vestiges de l'église. Il s'agit d'une vaste structure quadrangulaire composée de deux enclos, à l'intérieur desquels on relève une concentration de petites structures circulaires ou quadrangulaires (fig. 3). 


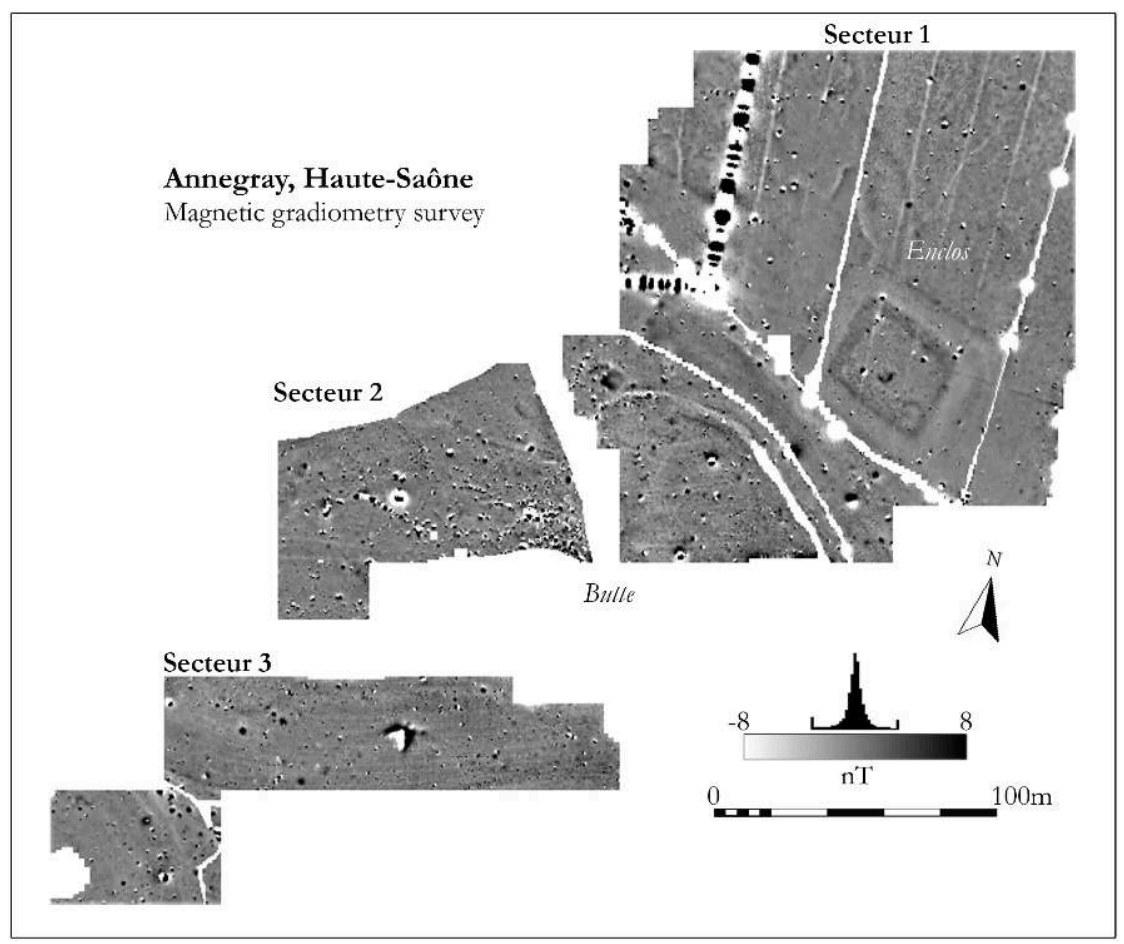

Fig. 3 - Annegray, magnétogramme des trois secteurs prospectés (R. Schot, université de Galway, 2010)

Le premier enclos est de plan quadrangulaire, avec des angles légèrement arrondis ; il est formé par un fossé dont la largeur serait comprise entre $\pm 6 \mathrm{~m}$ sur ses côtés nord et ouest et $\pm 2 \mathrm{~m}$ au sud et à l'est - les dimensions données à partir du magnétogramme doivent être prises avec précaution. Les côtés nord et ouest sont doublés par une bande plus mince pouvant correspondre à un talus. Le plan forme un quadrilatère, d'environ $40 \times 35 \mathrm{~m}$ orienté nord-est-sud-ouest, légèrement surélevé d'une cinquantaine de centimètres par rapport au champ. Le fossé taluté est entouré par une seconde clôture palissadée (?) distante de 7-8 $\mathrm{m}$ et mesurant $0,5 \mathrm{~m}$ à $1 \mathrm{~m}$ de largeur. Le petit côté sud-est est peu visible, mais cette seconde clôture mesure environ 65 x $50 \mathrm{~m}$. Des interruptions dans les angles nord-ouest et sud-ouest pourraient correspondre à des entrées, mais on n'enregistre aucune surface qui trahirait la présence d'une voie empierrée. On observe dans ce secteur des traces linéaires irrégulières pouvant correspondre à des levées de terre arasées (mesures magnétiques négatives). L'angle sud-est de l'enclos fossoyé est occupé par une construction circulaire d'environ $10 \mathrm{~m}$ de diamètre; d'autres vestiges de petites structures négatives linéaires ou ponctuelles (trous de poteaux?), de plan circulaire, se concentrent dans la moitié sud du premier enclos. Une structure semicirculaire, bien marquée, se distingue au centre de l'enclos fossoyé. Au nord des enclos, on relève la présence d'au moins trois structures circulaires (incomplètes), qui rappellent celles que l'on peut voir à l'œil nu sur le terrain, grâce aux anomalies de croissance du couvert végétal. L'escarpement, qui limite la butte accueillant les vestiges de l'église, est bien marqué par un tracé curviligne pouvant refléter un enrochement. On ne peut cependant pas déterminer si cette résonance magnétique négative est due à un aménagement anthropique ou à un affleurement rocheux. Mais l'absence de prolongement de cette hypothétique structure dans le champ voisin à l'ouest plaide en faveur d'un phénomène géologique. Trois autres structures potentielles, identifiées par 
des trous de poteaux, occuperaient le sommet de la butte et son flanc nord: la plus remarquable est un ensemble formé de deux structures circulaires emboîtées, dont le diamètre est compris entre $17 \mathrm{~m}$ pour la couronne extérieure et $5 \mathrm{~m}$ pour la couronne intérieure. Au pied de la butte, une structure est recouverte (recoupée ?) par une tranchée de réseau d'eau; elle se distingue par un plan rectangulaire d'environ $10 \times 7 \mathrm{~m}$ et une résonance négative, qui l'attribuerait plutôt à une construction en dur.

\section{Interprétation}

7 La découverte de cette structure à double enclos quadrangulaire est l'apport majeur de la première campagne de prospection géophysique à Annegray. Rappelons qu'elle se situe dans un terrain marécageux et donc, a priori, défavorable à un lotissement. Cette construction était tout à fait inattendue, même si, désormais, on peut la deviner sur les clichés aériens (fig. 1). La signature magnétique de la grande majorité des vestiges indique clairement que l'on est en présence de constructions où le bois et la terre sont prédominants. Quel que soit le degré d'exactitude de l'interprétation du magnétogramme, il est fortement vraisemblable qu'il s'agit d'un ensemble de constructions et d'aménagements recouvrant plusieurs phases chronologiques. Une rapide prospection pédestre n'a pas permis de recueillir le moindre mobilier, qui aurait pu donner un indice chronologique. C'est donc sur la base d'une comparaison typologique du plan des aménagements que l'on formulera des hypothèses sur la nature de l'occupation.

8 La première hypothèse est celle d'une Viereckschanze, enceinte quadrilatérale délimitée par un talus et un fossé, très répandue en Europe celtique et plutôt attribuée à la fin du second âge du fer ${ }^{5}$. Il pourrait aussi s'agir d'une structure funéraire de grande taille. Dans ce cas, on songe à un monument funéraire aristocratique, dont on connaît des exemples à la transition Hallstatt - la Tène, par exemple, dans la vallée de la Vanne, près de Troyes (Bourenton) ${ }^{6}$. Cependant, dans un cas comme dans l'autre, on est surpris par les dimensions peu communes du fossé de l'enclos intérieur ${ }^{7}$.

9 La seconde hypothèse serait celle d'une fortification médiévale en bois, qui pourrait alors être mise en relation avec l'émergence d'une petite seigneurie à Annegray. On sait peu de chose sur les seigneurs d'Annegray, au-delà de ce qu'en écrit (hasardeusement) Jules Vinot en $1886^{8}$. Selon lui, c'est à la fin du $x^{e}$ siècle qu'apparait cette famille placée sous la tutelle des Faucogney et qui aurait disposé d'un château, mais dont on ne connaît pas l'emplacement. En revanche, selon des études plus récentes, s'il est bien attesté qu'une famille a porté le nom d'Annegray aux XII ${ }^{e}$, $\mathrm{XII}^{\mathrm{e}}$ et $\mathrm{XVI}^{\mathrm{e}}$ siècles, on ne sait rien des liens qu'elle put avoir avec le hameau ${ }^{9}$ et on ignore même si elle possédait une résidence en ce lieu placé sous l'autorité du prieur. L'hypothèse d'une bâtisse médiévale en bois ne doit pourtant pas être exclue si l'on compare les plans et le mode de construction avec des maisons fortes connues ${ }^{10}$. Mais la localisation, l'absence de véritable tertre et de la moindre mention dans les sources écrites anciennes comme d'indices toponymiques ne plaident pas en faveur d'une construction médiévale.

10 La troisième hypothèse est celle qui recueille nos faveurs, même si elle n'a guère plus d'arguments déterminants que les précédentes, sinon historiographiques. Pourrait-il s'agir du castrum qu'évoque Jonas de Bobbio dans son hagiographie? Rappelons que Jonas relate des événements auxquels il n'a pas participé et décrit des lieux qu'il ne connaît pas obligatoirement. Dans ce cas, que signifie pour lui un ancien castrum ? La

Bulletin du centre d'études médiévales d'Auxerre | BUCEMA, 15 | 2011 
question se pose à Luxeuil, elle se pose également ici. Sous la plume d'un moine mérovingien, ce terme pourrait être générique et recouvrir des réalités architecturales très différentes d'un lieu à l'autre. Aussi, on connaît des enclos fossoyés et palissadés pour des petites forteresses routières ou des fortifications rurales de plaine de l'Antiquité tardive ${ }^{11}$. La comparaison est acceptable en termes de plans, de dimensions et de mode de construction, même si l'existence d'une voie antique n'est pas attestée archéologiquement. Se pourrait-il, dès lors, que les premiers moines irlandais aient réellement investi un ancien burgus gallo-romain? On reste étonné d'un dispositif défensif, où la palissade précède le fossé ${ }^{12}$, mais rien ne nous assure qu'ils sont contemporains. La création d'une seconde clôture - et le comblement du fossé ? pourrait marquer une évolution dans la nature de l'occupation du site. Une clôture monastique de plan quadrangulaire serait tout à fait inattendue dans ce contexte, mais répondrait à nos interrogations de départ sur les modalités de l'installation de Colomban. En outre, on notera que l'une des clôtures monastiques mérovingiennes les mieux documentées archéologiquement, à Hamage, est de plan quadrangulaire avec fossé et palissade ${ }^{13}$. La réoccupation éventuelle d'une petite fortification romaine par un monastère, à la fin $\mathrm{du} \mathrm{VI}^{\mathrm{e}}$ siècle, pourrait être mise en perspective avec le recensement opéré par S. Lebecq sur l'Angleterre ${ }^{14}$; les questions qu'il pose alors sur les motivations de ces choix d'implantations monastiques peuvent en effet être transposées à Annegray, en termes de peuplement, de cadre topographique ou de lieu lié à une "autorité du passé », ainsi que nous le pressentions pour ces fondations vosgiennes.

\section{Conclusion}

11 Dans l'état actuel des recherches, les résultats obtenus ne permettent pas d'aller audelà des hypothèses, même si celle d'un fortin romain ouvre des perspectives inédites et réjouissantes. Toutefois, pour sortir de ces spéculations, on ne pourra pas faire l'économie, à terme, d'une recherche plus approfondie passant par l'ouverture de sondages archéologiques après une nouvelle prospection radar-sol ou électrique concentrée sur les enclos. Seules les données archéologiques de terrain permettront de s'assurer de la chronologie et de la nature de l'occupation du site.

\section{NOTES}

1. G. CUGNIER, «La Voivre, lieu-dit Annegray. Site du monastère de saint Colomban ", Les premiers monuments chrétiens de la Gaule, t. 3, Paris, 1998, p. 141-143.

2. JONAS DE BOBBIO, Vitae Columbani abbatis discipulorumque eius, I, 6-7 (éd. cit., p. 72-74 ; trad. fr. A. DE VOGUÉ, JONAS DE BOBBIO,Vie de saint Colomban et de ses disciples, Bégrolles-en-Mauges, 1988, p. 112-113).

3. En remerciant Jacques Prudhon et Roger Dirand, de l'association des Amis de saint Colomban, pour ces informations. 
4. Projet intitulé : «Construction de l'espace au haut Moyen Âge dans le royaume burgonde : le cas de l'abbaye de Luxeuil et des monastères colombaniens ».

5. O. BUCHSENSCHUTZ et L. OLIVIER (dir.), Les viereckschanzen et les enceintes quadrilatérales en Europe celtique, Paris, 1989.

6. Les informations sur les viereckschangen et les monuments funéraires nous ont été transmises par P. Barral, que nous remercions.

7. Ce qui permet également d'exclure, a priori, une ferme gauloise.

8. J. VINOT, Les sires de Faucogney, vicomtes de Vesoul, Paris, 1886, p. 25.

9. SALSA. La Haute-Saône. Nouveau dictionnaire des communes, t. 6, 1974, p. 157-161.

10. On pourra comparer les structures ici décrites avec celles fouillées, par exemple, au château de Rouelbeau dans le canton de Genève ; J. TERRIER avec la coll. de M. JogUin REgELIN, « Rouelbeau : un château en bois édifié en 1318 au sommet d'un tertre artificiel », Archéologie suisse, 32/2 (2009), Bâle, p. 54-63 ; en Franche-Comté, un inventaire des maisons fortes, réalisé sous la direction de Jean-Jacques Schwien, a recensé des structures assez proches en plan de celle d'Annegray, comme à Cosges, Lavoncourt ou encore Lomont (en remerciant J.-J.Schwien pour ces informations).

11. M. Reddé, R. BRulet, R. Fellmann, J. K. HaAlebos et S. von SChnuRbein (dir.), L'architecture de la Gaule romaine, t. 1 (Les fortifications militaires),Bordeaux/Paris, 2006, p. 159-174.

12. Ce schéma, s'il reste rare, n'est pas complètement inconnu; c'est le cas notamment au monastère de Lisleagh: A. M. MoNK, «Early medieval secular and ecclesiastical settlement in munster ", Early medieval munster. Archaeology, history and society, Cork, 1998, p. 41.

13. É. LouIs, "Sorores ac fratres in Hamatico degentes. Naissance, évolution et disparition d'une abbaye au haut Moyen Âge : Hamage (France, Nord) », De la Meuse à l'Ardenne, 29 (1999), p. 17-47.

14. S. LEBECQ, «Monasterium constructum in castro quod lingua Anglorum Cnobheresburg vocatur (Bède, HEGA, III 19). De l'attraction exercée par les fortifications romaines sur les fondations monastiques dans l'Angleterre du très haut Moyen Âge ", in L'autorité du passé dans les sociétés médiévales, Rome, 2004, p. 277-295.

INDEX

Mots-clés : monastère, prospection géophysique

Index géographique : France/Annegray 